\title{
A profile of New Zealand 'Asian' participants of the 2008/09 Adult National Nutrition Survey: focus on dietary habits, nutrient intakes and health outcomes
}

\author{
Sherly M Parackal ${ }^{1, *}$, Claire Smith ${ }^{2}$ and Winsome Ruth Parnell ${ }^{2}$ \\ ${ }^{1}$ School of Population Health, Faculty of Medical and Health Science, The University of Auckland, Bldg 730, 261 \\ Morrin Road, Glen Innes, Pvt Bag 92019, Auckland, New Zealand: ${ }^{2}$ Department of Human Nutrition, University of \\ Otago, Dunedin, New Zealand
}

Submitted 29 November 2013: Final revision received 27 March 2014: Accepted 28 April 2014: First published online 2 June 2014

\begin{abstract}
Objective: To investigate similarities and differences in dietary habits, nutrient intakes and health outcomes of South Asians (SA) and East and South-East Asians (ESEA) and the New Zealand European and Other (NZEO) group, and to examine differences within 'Asian' subgroups according to duration of residence.

Design: Nutrient intake data from $24 \mathrm{~h}$ diet recalls and data from the dietary habits questionnaire, anthropometry and biochemical analyses from the cross-sectional 2008/09 Adult National Nutrition Survey in New Zealand were compared for participants categorized as SA, ESEA and NZEO.

Subjects: Adults aged 15 years and older ( $n$ 2995).

Setting: New Zealand households.

Results: SA were more likely to 'never' eat red meat in comparison to NZEO $(P<0.001)$ and among females also in comparison to ESEA $(P<0.05)$. Intakes of fats and some micronutrients (riboflavin, vitamin $\mathrm{B}_{6}, \mathrm{~B}_{12}$, Se) were lower among $\mathrm{SA}$ than NZEO $(P<0.05)$. Lower intakes of $\mathrm{Zn}$ and vitamin $\mathrm{B}_{12}$ were reported by SA females compared with ESEA and NZEO females $(P<0 \cdot 05)$. A higher percentage of SA were obese using ethnic-specific cut-offs, had lower indices of Fe status and reported diagnosed diabetes compared with NZEO and ESEA. Recent SA male migrants had higher intakes of $\beta$-carotene, vitamin $\mathrm{C}$ and $\mathrm{Ca}$ compared with long-term migrants $(P<0.05)$.

Conclusions: The results of the present study indicate that dietary habits, nutrient intakes, blood profile and body size differ significantly between Asian subgroups. It also provides some evidence for changes in dietary intakes according to duration of residence especially for SA males.
\end{abstract}

\author{
Keywords \\ South Asians \\ East/South-East Asians \\ Dietary habits \\ Nutrient intakes \\ Health outcomes \\ Duration of residence
}

In New Zealand (NZ) it is projected that by 2026, $15 \%$ of the population will be people of Asian ethnicity, a level similar to that projected for the Māori population, the indigenous people of $\mathrm{NZ}^{(1)}$. Nutrition-related health issues among 'Asian' immigrants to Western countries has been a topic of investigation in recent years ${ }^{(2-8)}$. Research has indicated an elevated risk for type 2 diabetes and CHD among South Asian immigrants ${ }^{(9-14)}$ and cancers among Chinese immigrants ${ }^{(15,16)}$.

Although migrants can be relatively healthier than the resident population on arrival, there is evidence that longer duration of residence can mitigate the healthy migrant status ${ }^{(17,18)}$. Numerous factors synergistically contribute towards this process, and changes in dietary patterns have been identified as an important independent risk factor for the development of chronic diseases among South Asian ${ }^{(5,14,19-21)}$ and Chinese ${ }^{(22)}$ migrants. The negative consequences of changed dietary patterns and acculturation have been observed among first-generation migrants $^{(23-25)}$ and among subsequent generations ${ }^{(26,27)}$.

There is a long history of Asian migration to NZ. However, few studies have reported dietary patterns and nutrient intakes of this growing sector. The 1977 National Diet Survey ${ }^{(28)}$, the 1989 Life in NZ survey ${ }^{(29)}$ and the 1997 National Nutrition Survey ${ }^{(30)}$ reported dietary intakes of adult New Zealanders. However, no attempt was made in these surveys to provide representative samples to report dietary intakes of Asians. In NZ the Diabetes Heart and Health Study was probably the first to report dietary intakes for Asian adults and indicated that Asians consumed more dietary 
cholesterol and less $\mathrm{Ca}$ and alcohol than Europeans ${ }^{(4)}$. However, reporting results for 'Asians' as an ethnic category is problematic, as they are a heterogeneous group. Combining them in a single 'Asian' category averages out subgroup differences and masks important distinctions ${ }^{(31)}$. The aims of the present paper were to profile 'Asian' participants of the 2008/09 Adult National Nutrition Survey (NZANS) and report dietary habits, nutrient intakes and health outcomes of Asian subgroups separately to gain some understanding of similarities and differences between these groups resident in NZ. It also aimed to report significant differences within the Asian subgroups according to duration of residence and compare the two Asian subgroups, South Asians (SA) and East and South-East Asians (ESEA), with the New Zealand European and Other (NZEO) group.

\section{Methods}

\section{Study design and sample}

The 2008/09 NZANS was a cross-sectional survey of NZ adults (15 years and older). A three-stage, stratified, probability-proportional-to-size sample design was used with an overall response rate of $61 \%(n$ 4721). To ensure adequate sample sizes to make comparisons, the Māori and Pacific ethnic groups and the age groups 15-18 years and 71 years and older were over-sampled. The study was conducted according to the guidelines laid down in the Declaration of Helsinki and all procedures involving human participants were approved by the Multi-Region Ethics Committee. Written informed consent was obtained from all participants. A computer-based interviewer-administered questionnaire was used to collect information on dietary habits, nutrition-related health and demographics. A full description of the methods is available elsewhere ${ }^{(32)}$.

\section{Demographic information and ethnicity}

Information on ethnicity was collected using the Statistics New Zealand standard ethnicity question. For the present research, if participants reported belonging to two or more groups they were assigned to a single group using the following order: (i) South Asian (SA); (ii) East and SouthEast Asian (ESEA); (iii) Māori; (iv) Pacific; and (v) New Zealand European and Others (NZEO). For example, if a participant reported he/she was Māori and East Asian he/ she was coded as East Asian. Data from participants coded as Māori and Pacific are not presented in this paper. Included in the SA group were participants who self-identified as Indian, Bangladeshi, Nepalese, Pakistani, Fiji Indian, Afghani and Sri Lankan; and in the ESEA group participants who reported they were Chinese (includes Malaysian, Hong Kong, Cambodian, Singaporean and Vietnamese Chinese), Malaysian, Taiwanese, Filipino, Cambodian, Vietnamese, Burmese, Indonesian, Thai, Japanese, Korean and Tibetan. The 'Others' in the NZEO group includes all other ethnicities mainly Latin American and African.
Participants reported their highest school-level qualification, and if applicable their highest post-school qualification (greater than 3 months' study or training). Four groups were derived: (i) no school or post-school qualifications; (ii) school qualifications only; (iii) trade or technical qualifications; and (iv) post-school qualifications (professional or tertiary).

The NZ Index of Deprivation (NZDep06) was used to measure socio-economic status. This is an area-based measure and reflects eight dimensions of material deprivation $^{(33)}$. For the purposes of the 2008/09 NZANS deprivation scores were divided into quintiles.

\section{Duration of residence}

Participants who reported they were not born in NZ were asked the year they arrived in NZ. Duration of residence was categorized into a dichotomous variable: (i) $<10$ years of residence (recent migrants); and (ii) $\geq 10$ years of residence and NZ born (long-term migrants). Statistically significant differences in dietary habits and nutrient intakes according to duration of residence have been reported in the text but are not included in the respective tables.

\section{Dietary habits}

Participants were asked about their frequency of consumption of selected foods in the 'past four weeks'.

\section{Nutrient intakes}

A four-stage $24 \mathrm{~h}$ diet recall was administered by trained interviewers using LINZ24 (a computer software system) ${ }^{(32)}$. Of those completing one diet recall a randomly selected sub-sample (25\%) also completed a second $24 \mathrm{~h}$ diet recall; however, mean nutrient intakes were calculated from the first day of recall only because of inadequate numbers of repeat recalls in the Asian groups. Energy and nutrient intakes were determined using a tailored and expanded version of the New Zealand Food Composition Database ${ }^{(32)}$.

\section{Biochemical/blood indices}

A non-fasting venous blood sample was provided by a total of 3348 participants (not pregnant) and 3315 provided a spot urine sample. Full details on the analytical methods for all biochemical analyses are available elsewhere ${ }^{(32)}$. Concentrations of glycated $\mathrm{Hb}$ (HbA1c), total cholesterol, HDL-cholesterol, serum ferritin, $\mathrm{Hb}$, serum Fe, and red blood cell (RBC) and serum folate were reported.

\section{Iron and folate status}

Low Hb was defined as: < 136 g/l (15-19 years, males); $<137 \mathrm{~g} / 1$ (20-49 years, males); <133 g/1 (50-69 years, males); <124 g/l (70+ years, males); <120 g/1 (15-69 years, females); and $<118 \mathrm{~g} / 1$ ( $70+$ years, females). Fe deficiency with or without anaemia was defined as serum ferritin $<12 \mu \mathrm{g} / \mathrm{l}$ and $\mathrm{Zn}$ protoporphyrin $>60 \mu \mathrm{mol} / \mathrm{mol}$. Fe deficiency with anaemia was defined as serum ferritin $<12 \mu \mathrm{g} / \mathrm{l}$ and $\mathrm{Zn}$ protoporphyrin $>60 \mu \mathrm{mol} / \mathrm{mol}$ and low $\mathrm{Hb}$. 
Low RBC folate was defined as $<317 \mathrm{nmol} / 1$ and low serum folate as $<6.8 \mathrm{mmol} / \mathrm{l}$.

\section{Blood pressure}

Diastolic and systolic blood pressures were measured on non-pregnant participants by trained interviewers using an OMRON HEM907 instrument. Three measurements were taken and the mean of the second and third measurements was used.

\section{Diagnosed diabetes}

Participants were asked 'Have you ever been told by a doctor that you have diabetes?' Those answering affirmatively were categorized as having 'doctor diagnosed diabetes'.

\section{Anthropometric measurements}

Two measurements for height and weight were made for each participant. If the measurements differed by $1 \%$, then a third measurement was taken. WHO BMI cutoffs were used to classify participants over 19 years of age into underweight $\left(\mathrm{BMI}<18.5 \mathrm{~kg} / \mathrm{m}^{2}\right)$, normal $(\mathrm{BMI}=$ $\left.18.5-24.99 \mathrm{~kg} / \mathrm{m}^{2}\right)$, overweight $\left(\mathrm{BMI}=25.0-29.99 \mathrm{~kg} / \mathrm{m}^{2}\right)$ and obese (BMI $\left.\geq 30 \cdot 0 \mathrm{~kg} / \mathrm{m}^{2}\right)^{(34)}$. Due to small numbers the underweight category was collapsed into the normal category (BMI $<25.0 \mathrm{~kg} / \mathrm{m}^{2}$ ). Sex- and age-specific cut-offs were used for participants aged $15-18$ years ${ }^{(35)}$.

The prevalence of overweight and obesity were also defined using ethnic-specific cut-offs ${ }^{(36)}$ as evidence indicates obesity is under-diagnosed using standard WHO guidelines among 'Asian' people ${ }^{(37)}$. For SA and ESEA participants over 19 years the following cut-offs were used: normal $\left(\mathrm{BMI}<23.0 \mathrm{~kg} / \mathrm{m}^{2}\right)$, overweight $(\mathrm{BMI}=23 \cdot 0-24.99$ $\mathrm{kg} / \mathrm{m}^{2}$ ) and obese $\left(\mathrm{BMI} \geq 25 \cdot 0 \mathrm{~kg} / \mathrm{m}^{2}\right)$. To define overweight and obesity in Asian participants aged 15-18 years, sex- and age-specific cut-offs were used ${ }^{(38)}$.

\section{Statistical analysis}

For all analyses survey commands were used to allow for the complex survey design, including sampling weights and primary sampling units. Results reported were weighted to reflect population estimates. Separate analyses were conducted for males and females. The $\chi^{2}$ test was used to examine the associations between categorical dietary habit variables and ethnicity.

Linear regression was used to compare nutrient intakes by ethnicity, with age as a covariate. Nutrient intakes (apart from percentage of energy) were positively skewed and therefore were transformed using the natural $\log$ transformation to normalize them. Therefore geometric means are reported for all nutrients apart from percentage of total energy from fat, saturated fat, polyunsaturated fat, monounsaturated fat, carbohydrate and protein. Linear regression with adjustment for age was used to compare serum total cholesterol, HDLcholesterol, $\mathrm{Hb}$, ferritin, serum folate, RBC folate, HbA1c and blood pressure. Logistic regression, with adjustment for age, was used to examine the relationship between dichotomous blood and health indices, overweight, obesity and ethnic group. For ESEA males there were no observations for low Fe stores $(<12 \mu \mathrm{g} / \mathrm{l})$, Fe deficiency, Fe-deficiency anaemia, low serum folate $(<6.8 \mathrm{mmol} / \mathrm{l})$, high HbA1c $(\geq 6.5 \%)$ and diagnosed diabetes; therefore a comparison could not be made with other ethnic groups using logistic regression. Similarly for ESEA females there were no observations for low RBC folate $(<317 \mathrm{nmol} / \mathrm{l})$.

For nutrient intakes and dietary habits, analyses were repeated for SA and ESEA with comparison by duration of residence. All statistical analyses were conducted using the Stata statistical software package version 12. All statistical tests were two-sided and $P<0.05$ was considered statistically significant in all cases.

\section{Results}

The percentage of 'Asian' participants (9.8\%) was comparable to that in the NZ population, based on the estimates for 2006 by Statistics New Zealand (11\%) and calculated by excluding Māori and Pacific Peoples ${ }^{(39)}$. More ESEA and SA were younger compared with NZEO $(P<0 \cdot 001)$. Although ESEA and SA were more likely to have higher educational qualifications than NZEO $(P<0 \cdot 001)$, the percentage of 'Asians', especially SA, living in deprived areas was high $(P=0.003)$. The majority of 'Asian' participants were migrants who had lived in NZ for less than 10 years (i.e. recent migrants; $P<0 \cdot 001$; Table 1 ).

\section{Dietary habits}

There were significant differences between ethnic groups with respect to meeting the dietary guidelines for fruits and vegetables, bread consumption, milk consumption, meat consumption and preparation, and alcohol use (Table 2).

In particular, meeting the dietary guidelines for fruit and vegetables ( $5+$ a day) was low for ESEA males ( $9 \%)$ in comparison to SA $(32 \% ; P=0.011)$ and NZEO ( $40 \%$; $P=0.000)$ males; and for SA females $(15 \%)$ in comparison to ESEA $(35 \% ; P<0.05)$ and NZEO $(55 \% ; P<0.001)$ females.

While ESEA males were the group most likely to eat chicken 'three or more times per week', SA females were the group most likely to 'never' eat chicken (26\%), red meat $(25 \%)$ and processed meat $(55 \% ; P<0.05$; Table 2$)$. Among males, SA males were the group who most often 'never' ate red meat (21\%). Among males, both SA and ESEA were more likely to 'never' eat processed meat compared with NZEO males $(P<0 \cdot 05$; Table 2$)$. Among males, recent SA migrants were more likely to 'never' consume processed meat (60.2\%; $95 \%$ CI 44.3, $76 \cdot 2 \%)$ compared with long-term migrants (26.5\%; $95 \%$ CI 5.0, $47.8 \% ; P=0.009)$.

With respect to removing fat from meat and from chicken, SA males and females were more likely to do this compared with NZEO males and females $(P<0 \cdot 05$; Table 2$)$. 
Table 1 Sample characteristics by ethnicity $\left(n\right.$ 2995) ${ }^{\star}$, 2008/09 Adult National Nutrition Survey, New Zealand

\begin{tabular}{|c|c|c|c|c|c|c|c|}
\hline \multirow[b]{3}{*}{ Sample characteristic } & \multicolumn{6}{|c|}{ Ethnicity } & \multirow[b]{3}{*}{$P$ value $\neq$} \\
\hline & \multicolumn{2}{|c|}{ SA } & \multicolumn{2}{|c|}{ ESEA } & \multicolumn{2}{|c|}{ NZEO } & \\
\hline & $n$ & $\% \dagger$ & $n$ & $\% \dagger$ & $n$ & $\% \dagger$ & \\
\hline Gender & & & & & & & 0.229 \\
\hline Male & 64 & $57 \cdot 0$ & 57 & 43.2 & 1187 & 47.4 & \\
\hline Female & 66 & $43 \cdot 0$ & 80 & $56 \cdot 8$ & 1541 & $52 \cdot 6$ & \\
\hline Age group & & & & & & & $<0.001$ \\
\hline $15-18$ years & 33 & $6 \cdot 8$ & 41 & 8.2 & 451 & $6 \cdot 8$ & \\
\hline $19-30$ years & 25 & $28 \cdot 6$ & 38 & 44.6 & 283 & $17 \cdot 6$ & \\
\hline $31-50$ years & 43 & $40 \cdot 6$ & 35 & 31.3 & 595 & 35.5 & \\
\hline $51-70$ years & 23 & $22 \cdot 9$ & 14 & 13.7 & 524 & $28 \cdot 8$ & \\
\hline $71+$ years & 6 & 1.2 & 9 & 2.0 & 875 & 11.4 & \\
\hline Qualifications & & & & & & & $<0.001$ \\
\hline No school or post-school qualifications & 24 & $7 \cdot 7$ & 16 & 5.5 & 696 & $18 \cdot 7$ & \\
\hline School qualifications only & 37 & $20 \cdot 7$ & 58 & $30 \cdot 8$ & 798 & $26 \cdot 6$ & \\
\hline Trade or technical qualifications & 21 & 21.5 & 11 & $5 \cdot 8$ & 550 & 23.5 & \\
\hline Post-school qualifications (professional or tertiary) & 46 & $50 \cdot 2$ & 52 & $58 \cdot 1$ & 674 & $31 \cdot 2$ & \\
\hline NZDep06 quintile§ & & & & & & & 0.003 \\
\hline I (low deprivation) & 12 & $15 \cdot 6$ & 23 & $15 \cdot 1$ & 509 & $22 \cdot 6$ & \\
\hline II & 19 & $10 \cdot 9$ & 28 & 23.2 & 605 & 23.2 & \\
\hline III & 19 & $18 \cdot 1$ & 31 & 31.0 & 514 & 21.9 & \\
\hline IV & 36 & $20 \cdot 5$ & 28 & $14 \cdot 3$ & 605 & $19 \cdot 0$ & \\
\hline V (high deprivation) & 44 & 34.9 & 27 & $16 \cdot 5$ & 495 & $13 \cdot 1$ & \\
\hline Duration of residence by gender & & & & & & & 0.000 \\
\hline Recent migrants & & & & & & & \\
\hline Males & 37 & $60 \cdot 3$ & 50 & 71.5 & 29 & $2 \cdot 4$ & \\
\hline Females & 37 & $59 \cdot 8$ & 32 & 58.5 & 29 & 4.0 & \\
\hline Long-term migrants & & & & & & & \\
\hline Males & 28 & $39 \cdot 8$ & 30 & 28.5 & 1504 & $97 \cdot 6$ & \\
\hline Females & 26 & $40 \cdot 1$ & 25 & 41.5 & 1155 & $96 \cdot 0$ & \\
\hline
\end{tabular}

SA, South Asians; ESEA; East and South-East Asians; NZEO, New Zealand Europeans and Others.

*Number of those with $24 \mathrm{~h}$ diet recall data (excluding Pacific and Māori participants).

†Weighted percentage.

$\ddagger P$ value from $\chi^{2}$ test.

$\S N Z D e p 06,2006$ New Zealand Index of Deprivation, is an area-based measure of deprivation.

Fast food ('more than three times per week') was reported by $19 \%$ of SA males compared with $7 \%$ of NZEO males. In contrast among females, the SA ethnic group seldom consumed fast food ( $P=0 \cdot 014$; Table 2$)$.

\section{Nutrient intakes}

Age-adjusted mean nutrient intakes are reported by sex in Table 3. SA males had lower intakes of energy, fat, saturated fat (all $P<0.05)$ and percentage of energy from fat $(P<0.05)$ and saturated fat $(P<0.001)$, and higher percentage of energy from carbohydrate $(P<0.05)$, compared with NZEO males. Cholesterol intake was also lower compared with NZEO $(P<0 \cdot 01)$. Similarly, SA females had lower intakes of energy $(P<0.01)$, protein $(P<0.001)$, fat $(P<0 \cdot 01)$, monounsaturated fat $(P<0 \cdot 001)$ and higher percentage of energy from carbohydrate $(P<0 \cdot 01)$ compared with NZEO females. SA females also had lower energy $(P<0 \cdot 05)$, protein $(P<0.01)$ and monounsaturated fat $(P<0.05)$ intakes compared with ESEA females. Males $(P<0.001)$ and females $(P=0.003)$ of both Asian subgroups had lower intakes of saturated fats compared with NZEO. Both ESEA males and females had higher percentage of energy from protein $(P<0.05)$ and carbohydrate $(P<0.05)$ compared with NZEO (Table 3).
Both SA males and females reported lower intakes of vitamin $\mathrm{B}_{12}$, Se, niacin and retinol compared with NZEO $(P<0.05)$. In addition, SA males reported lower intakes of $\mathrm{Ca}$, riboflavin, vitamin $\mathrm{B}_{6}$ and vitamin $\mathrm{A}$ compared with NZEO males $(P<0 \cdot 05)$. SA females reported lower intakes of $\mathrm{K}$ and $\mathrm{Zn}$ compared with NZEO females $(P<0.05)$. Compared with ESEA, mean vitamin $\mathrm{B}_{12}$, Se, $\mathrm{Zn}$ (all $P<0 \cdot 001)$ and niacin intakes $(P<0 \cdot 01)$ were lower for SA females. The micronutrient intake of ESEA males and females was also lower compared with NZEO for retinol, thiamin and $\mathrm{Ca}(P<0.05$; Table 3$)$. Furthermore, mean intakes of vitamin $\mathrm{A}(P<0.001)$ and riboflavin $(P<0.05)$ were lower for ESEA males compared with NZEO males.

Recent SA male migrants had higher intakes of carbohydrate (288 g v. $234 \mathrm{~g} ; P=0 \cdot 036$ ), dietary fibre (21 g $v$. $17 \mathrm{~g} ; P=0.008)$, retinol $(225 \mu \mathrm{g} v .133 \mu \mathrm{g} ; P=0.027)$ and $\mathrm{Ca}$ (690 v. $492 \mathrm{mg} ; P=0 \cdot 012$ ) than long-term migrants.

\section{Biochemical and blood indices}

There was no difference in the levels of serum total cholesterol and HDL-cholesterol among males and females (Table 4). In general, indices of Fe status were lower for SA compared with NZEO. A higher percentage of SA males had low Fe stores (17\%), were Fe deficient $(12.7 \%)$ or were 
Table 2 Dietary habits and alcohol consumption by gender and ethnicity ( $n$ 2995), 2008/09 Adult National Nutrition Survey, New Zealand

\begin{tabular}{|c|c|c|c|c|c|c|c|c|}
\hline \multirow[b]{2}{*}{ Dietary habit } & \multirow[b]{2}{*}{ Sex } & \multicolumn{2}{|c|}{$\mathrm{SA}(n 130)$} & \multicolumn{2}{|c|}{ ESEA $(n 137)$} & \multicolumn{2}{|c|}{ NZEO ( $n$ 2728) } & \multirow[b]{2}{*}{$P$ value* } \\
\hline & & $\%$ & $95 \% \mathrm{Cl}$ & $\%$ & $95 \% \mathrm{Cl}$ & $\%$ & $95 \% \mathrm{Cl}$ & \\
\hline \multicolumn{9}{|l|}{ Fruit and vegetable servings per day } \\
\hline $5+$ a day & $\begin{array}{c}\mathrm{M} \\
\mathrm{F}\end{array}$ & $\begin{array}{l}31 \cdot 6 \\
15 \cdot 4\end{array}$ & $\begin{array}{r}18 \cdot 5,48 \cdot 3 \\
7 \cdot 9,27 \cdot 8\end{array}$ & $\begin{array}{l}8 \cdot 7 \S, \| \\
34.6\end{array}$ & $\begin{array}{r}3 \cdot 1,22 \cdot 0 \\
21 \cdot 6,50 \cdot 4\end{array}$ & $\begin{array}{l}39 \cdot 7 \\
55 \cdot 3\end{array}$ & $\begin{array}{l}35 \cdot 9,43 \cdot 6 \\
51 \cdot 8,58 \cdot 7\end{array}$ & $\begin{array}{l}<0.001 \\
<0.001\end{array}$ \\
\hline \multicolumn{9}{|l|}{ Slices of bread consumed } \\
\hline Never or less than 1 slice/d & $\begin{array}{l}M \\
F\end{array}$ & $\begin{array}{l}22 \cdot 7 \S \\
8 \cdot 1 \S, \|\end{array}$ & $\begin{array}{r}9 \cdot 6,35 \cdot 7 \\
13 \cdot 2,15 \cdot 0\end{array}$ & $\begin{array}{l}37.0 \S \\
29.8\end{array}$ & $\begin{array}{l}19 \cdot 4,54 \cdot 5 \\
16 \cdot 6,43 \cdot 1\end{array}$ & $\begin{array}{r}8 \cdot 2 \\
17 \cdot 0\end{array}$ & $\begin{array}{r}5.9,10.5 \\
14.3,19.7\end{array}$ & $\begin{array}{r}<0.001 \\
0.051\end{array}$ \\
\hline 1-4 slices/d & $\begin{array}{l}\mathrm{M} \\
\mathrm{F}\end{array}$ & $\begin{array}{l}61.2 \\
77 \cdot 5\end{array}$ & $\begin{array}{l}46 \cdot 5,74 \cdot 1 \\
59 \cdot 6,89 \cdot 0\end{array}$ & $\begin{array}{l}54 \cdot 4 \\
65 \cdot 1\end{array}$ & $\begin{array}{l}37 \cdot 8,70 \cdot 1 \\
50 \cdot 3,77 \cdot 4\end{array}$ & $\begin{array}{l}68 \cdot 7 \\
77 \cdot 0\end{array}$ & $\begin{array}{l}64 \cdot 6,72 \cdot 5 \\
73 \cdot 9,79 \cdot 8\end{array}$ & \\
\hline More than 4 slices/d & $\begin{array}{l}\mathrm{M} \\
\mathrm{F}\end{array}$ & $\begin{array}{l}16 \cdot 2 \\
14 \cdot 3\end{array}$ & $\begin{array}{r}8.0,30 \cdot 0 \\
5 \cdot 1,34 \cdot 4\end{array}$ & $\begin{array}{l}8.6 \\
5.1\end{array}$ & $\begin{array}{r}2 \cdot 6,24 \cdot 6 \\
1 \cdot 3,18.7\end{array}$ & $\begin{array}{r}23 \cdot 1 \\
6.0\end{array}$ & $\begin{array}{c}19 \cdot 8,26 \cdot 8 \\
4.6,7.9\end{array}$ & \\
\hline \multicolumn{9}{|l|}{ Milk consumption } \\
\hline Never & $\begin{array}{l}M \\
F\end{array}$ & $\begin{array}{l}0 \S, \| \\
0.8\end{array}$ & $0.2,3.3$ & $\begin{array}{r}2.6 \\
10 \cdot 2\end{array}$ & $\begin{array}{l}0 \cdot 8,8 \cdot 1 \\
4 \cdot 1,23 \cdot 1\end{array}$ & $\begin{array}{l}5 \cdot 1 \\
5 \cdot 6\end{array}$ & $\begin{array}{l}3 \cdot 5,7 \cdot 3 \\
4 \cdot 1,7 \cdot 4\end{array}$ & $\begin{array}{r}<0.001 \\
0.082\end{array}$ \\
\hline Whole & $\begin{array}{l}M \\
F\end{array}$ & $\begin{array}{l}63.8 \\
44.9\end{array}$ & $\begin{array}{l}47 \cdot 8,77 \cdot 3 \\
28 \cdot 8,62 \cdot 2\end{array}$ & $\begin{array}{l}38.6 \\
40 \cdot 2\end{array}$ & $\begin{array}{l}22 \cdot 1,58 \cdot 1 \\
27 \cdot 6,54 \cdot 2\end{array}$ & $\begin{array}{l}44 \cdot 1 \\
34 \cdot 0\end{array}$ & $\begin{array}{l}40 \cdot 3,47 \cdot 9 \\
30 \cdot 6,37 \cdot 6\end{array}$ & \\
\hline Reduced fat & $\begin{array}{l}\mathrm{M} \\
\mathrm{F}\end{array}$ & $\begin{array}{l}36 \cdot 2 \\
51 \cdot 4\end{array}$ & $\begin{array}{l}22 \cdot 7,52 \cdot 2 \\
34 \cdot 4,68 \cdot 2\end{array}$ & $\begin{array}{l}48 \cdot 6 \\
40 \cdot 4\end{array}$ & $\begin{array}{l}30 \cdot 4,67 \cdot 2 \\
27 \cdot 7,54 \cdot 5\end{array}$ & $\begin{array}{l}49 \cdot 0 \\
56 \cdot 1\end{array}$ & $\begin{array}{l}45 \cdot 3,52 \cdot 8 \\
52 \cdot 1,60 \cdot 0\end{array}$ & \\
\hline Other† & $\begin{array}{l}\mathrm{M} \\
\mathrm{F}\end{array}$ & $\begin{array}{l}0 \\
2 \cdot 8\end{array}$ & $0 .-11.6$ & $\begin{array}{r}10 \cdot 3 \\
9 \cdot 3\end{array}$ & $\begin{array}{l}3 \cdot 1,28 \cdot 9 \\
3 \cdot 7,21 \cdot 4\end{array}$ & $\begin{array}{l}1 \cdot 8 \\
4 \cdot 4\end{array}$ & $\begin{array}{l}1 \cdot 0,3 \cdot 2 \\
3 \cdot 1,6 \cdot 1\end{array}$ & \\
\hline \multicolumn{9}{|l|}{ Frequency of eating chicken } \\
\hline Never & $\begin{array}{l}M \\
F\end{array}$ & $\begin{array}{l}11.5 \\
25 \cdot 6 \S, \|\end{array}$ & $\begin{array}{r}4 \cdot 2,27 \cdot 5 \\
12 \cdot 6,45 \cdot 1\end{array}$ & $\begin{array}{l}0.6 \S, \| \\
7.5\end{array}$ & $\begin{array}{l}0 \cdot 1,4 \cdot 1 \\
2 \cdot 6,19 \cdot 7\end{array}$ & $\begin{array}{l}7 \cdot 3 \\
5 \cdot 0\end{array}$ & $\begin{array}{l}5 \cdot 6,9 \cdot 4 \\
4 \cdot 0,6 \cdot 4\end{array}$ & $\begin{array}{r}0.005 \\
<0.001\end{array}$ \\
\hline Two times or less per week & $\begin{array}{l}\mathrm{M} \\
\mathrm{F}\end{array}$ & $\begin{array}{l}49.9 \S \\
47.4 \S\end{array}$ & $\begin{array}{l}35 \cdot 7,64 \cdot 1 \\
30 \cdot 3,65 \cdot 2\end{array}$ & $\begin{array}{l}56 \cdot 8 \\
56 \cdot 3\end{array}$ & $\begin{array}{l}39 \cdot 6,72 \cdot 5 \\
41 \cdot 5,70 \cdot 1\end{array}$ & $\begin{array}{l}69 \cdot 1 \\
66 \cdot 9\end{array}$ & $\begin{array}{l}65 \cdot 3,72 \cdot 6 \\
63 \cdot 6,70 \cdot 2\end{array}$ & \\
\hline Three or more times per week & $\begin{array}{l}\mathrm{M} \\
\mathrm{F}\end{array}$ & $\begin{array}{l}37.3 \\
31.4\end{array}$ & $\begin{array}{l}16 \cdot 9,57 \cdot 8 \\
10 \cdot 4,52 \cdot 5\end{array}$ & $\begin{array}{l}42 \cdot 7 \S \\
35 \cdot 4\end{array}$ & $\begin{array}{l}32 \cdot 7,79 \cdot 4 \\
19 \cdot 1,51 \cdot 8\end{array}$ & $\begin{array}{l}25 \cdot 0 \\
27 \cdot 0\end{array}$ & $\begin{array}{l}21 \cdot 0,29 \cdot 0 \\
23 \cdot 5,30 \cdot 5\end{array}$ & \\
\hline \multicolumn{9}{|l|}{ Frequency of eating red meat } \\
\hline Never & $\begin{array}{l}M \\
F\end{array}$ & $\begin{array}{l}21 \cdot 1 \S \\
42 \cdot 7 \S, \|\end{array}$ & $\begin{array}{l}11 \cdot 2,36 \cdot 3 \\
26 \cdot 5,60 \cdot 6\end{array}$ & $\begin{array}{l}6 \cdot 5 \\
8 \cdot 1\end{array}$ & $\begin{array}{l}1 \cdot 7,21 \cdot 6 \\
3 \cdot 1,19 \cdot 6\end{array}$ & $\begin{array}{l}3 \cdot 2 \\
4.9\end{array}$ & $\begin{array}{l}2 \cdot 1,4 \cdot 8 \\
3 \cdot 6,6 \cdot 7\end{array}$ & $\begin{array}{l}<0.001 \\
<0.001\end{array}$ \\
\hline Two times or less per week & $\begin{array}{l}M \\
F\end{array}$ & $\begin{array}{l}40.5 \S \\
42.2\end{array}$ & $\begin{array}{l}27 \cdot 7,54 \cdot 7 \\
26 \cdot 4,59 \cdot 6\end{array}$ & $\begin{array}{l}31 \cdot 3 \\
39 \cdot 2\end{array}$ & $\begin{array}{l}17 \cdot 2,49 \cdot 9 \\
27 \cdot 1,52 \cdot 9\end{array}$ & $\begin{array}{l}31 \cdot 2 \\
36 \cdot 1\end{array}$ & $\begin{array}{l}27 \cdot 7,34 \cdot 9 \\
32 \cdot 8,39 \cdot 6\end{array}$ & \\
\hline Three or more times per week & $\begin{array}{l}\mathrm{M} \\
\mathrm{F}\end{array}$ & $\begin{array}{l}32 \cdot 3 \S, \| \\
15 \cdot 2 \S, \|\end{array}$ & $\begin{array}{r}12 \cdot 6,52 \cdot 0 \\
3.5,26 \cdot 8\end{array}$ & $\begin{array}{l}68 \cdot 9 \\
52 \cdot 7\end{array}$ & $\begin{array}{l}46 \cdot 8,91 \cdot 0 \\
39 \cdot 4,65 \cdot 9\end{array}$ & $\begin{array}{l}65.9 \\
59.0\end{array}$ & $\begin{array}{l}61 \cdot 6,70 \cdot 2 \\
55 \cdot 4,62 \cdot 5\end{array}$ & \\
\hline \multicolumn{9}{|l|}{ Frequency of eating processed meat } \\
\hline Never & $\begin{array}{l}M \\
F\end{array}$ & $\begin{array}{l}46 \cdot 7 \S \\
54 \cdot 7 \S, \|\end{array}$ & $\begin{array}{l}32 \cdot 0,61 \cdot 9 \\
36 \cdot 9,71 \cdot 3\end{array}$ & $\begin{array}{l}30 \cdot 3 \S \\
20 \cdot 5\end{array}$ & $\begin{array}{l}14 \cdot 9,51 \cdot 8 \\
11 \cdot 9,33 \cdot 1\end{array}$ & $\begin{array}{r}6 \cdot 4 \\
12 \cdot 3\end{array}$ & $\begin{array}{c}4 \cdot 8,8 \cdot 6 \\
10 \cdot 2,14 \cdot 8\end{array}$ & $\begin{array}{l}<0.001 \\
<0.001\end{array}$ \\
\hline Two times or less per week & $\begin{array}{l}\mathrm{M} \\
\mathrm{F}\end{array}$ & $\begin{array}{l}46 \cdot 2 \\
38 \cdot 0 \S, \|\end{array}$ & $\begin{array}{l}30 \cdot 9,62 \cdot 2 \\
22 \cdot 3,56 \cdot 7\end{array}$ & $\begin{array}{l}32.8 \\
64.6\end{array}$ & $\begin{array}{l}17 \cdot 6,52 \cdot 7 \\
50 \cdot 4,76 \cdot 6\end{array}$ & $\begin{array}{l}57 \cdot 3 \\
62 \cdot 8\end{array}$ & $\begin{array}{l}53 \cdot 3,61 \cdot 1 \\
59 \cdot 2,66 \cdot 2\end{array}$ & \\
\hline Three or more times per week & $\begin{array}{l}M \\
F\end{array}$ & $\begin{array}{l}7 \cdot 1 \S, \| \\
7 \cdot 4 \S, \|\end{array}$ & $\begin{array}{r}0,14.9 \\
0.1,14.6\end{array}$ & $\begin{array}{l}36.9 \\
14.9\end{array}$ & $\begin{array}{r}18 \cdot 5,55 \cdot 3 \\
4.8,35 \cdot 0\end{array}$ & $\begin{array}{l}36 \cdot 3 \\
24 \cdot 9\end{array}$ & $\begin{array}{l}32 \cdot 5,40 \cdot 0 \\
21 \cdot 8,28 \cdot 1\end{array}$ & \\
\hline \multicolumn{9}{|l|}{ Frequency of eating fresh/frozen/canned fish/seafood } \\
\hline Never & $\begin{array}{l}M \\
F\end{array}$ & $\begin{array}{l}23 \cdot 4 \\
33 \cdot 3\end{array}$ & $\begin{array}{l}12 \cdot 8,38.9 \\
18 \cdot 8,51 \cdot 9\end{array}$ & $\begin{array}{l}12 \cdot 4 \\
5 \cdot 8 \S, \|\end{array}$ & $\begin{array}{l}3 \cdot 6,34 \cdot 7 \\
2 \cdot 2,14 \cdot 4\end{array}$ & $\begin{array}{l}24.9 \\
22.9\end{array}$ & $\begin{array}{l}21 \cdot 4,28 \cdot 7 \\
20 \cdot 1,26 \cdot 1\end{array}$ & $\begin{array}{l}0.247 \\
0.002\end{array}$ \\
\hline Less than 1 time per week & $\begin{array}{l}\mathrm{M} \\
\mathrm{F}\end{array}$ & $\begin{array}{l}37 \cdot 1 \\
26 \cdot 9\end{array}$ & $\begin{array}{l}22 \cdot 4,54 \cdot 7 \\
14 \cdot 4,44 \cdot 6\end{array}$ & $\begin{array}{l}49 \cdot 2 \\
66 \cdot 8\end{array}$ & $\begin{array}{l}32 \cdot 0,66 \cdot 5 \\
53 \cdot 5,77 \cdot 8\end{array}$ & $\begin{array}{l}48 \cdot 9 \\
49 \cdot 1\end{array}$ & $\begin{array}{l}45 \cdot 0,52 \cdot 9 \\
45 \cdot 5,52 \cdot 8\end{array}$ & \\
\hline Once or more per week & $\begin{array}{l}\mathrm{M} \\
\mathrm{F}\end{array}$ & $\begin{array}{l}39.5 \\
39.8\end{array}$ & $\begin{array}{l}22 \cdot 9,58 \cdot 9 \\
23 \cdot 8,58 \cdot 3\end{array}$ & $\begin{array}{l}38 \cdot 4 \\
27 \cdot 4\end{array}$ & $\begin{array}{l}22 \cdot 4,57 \cdot 3 \\
17 \cdot 3,40 \cdot 5\end{array}$ & $\begin{array}{l}26 \cdot 2 \\
28 \cdot 0\end{array}$ & $\begin{array}{l}22 \cdot 9,29 \cdot 8 \\
24 \cdot 7,31 \cdot 4\end{array}$ & \\
\hline \multicolumn{9}{|l|}{ Other dietary habits } \\
\hline Add salt to food regularly/always & $\begin{array}{l}M \\
F\end{array}$ & $\begin{array}{l}38.5 \\
44.5\end{array}$ & $\begin{array}{l}24 \cdot 5,54 \cdot 8 \\
27 \cdot 8,62 \cdot 5\end{array}$ & $\begin{array}{l}41.4 \\
34.6\end{array}$ & $\begin{array}{l}25 \cdot 0,60 \cdot 0 \\
21 \cdot 0,51 \cdot 3\end{array}$ & $\begin{array}{l}33 \cdot 7 \\
47 \cdot 4\end{array}$ & $\begin{array}{l}29 \cdot 9,37 \cdot 8 \\
43 \cdot 8,51 \cdot 0\end{array}$ & $\begin{array}{l}0.587 \\
0.266\end{array}$ \\
\hline Choose reduced salt varieties regularly/always & $\begin{array}{c}\mathrm{M} \\
\mathrm{F}\end{array}$ & $\begin{array}{l}5 \cdot 7 \\
5 \cdot 4\end{array}$ & $\begin{array}{l}2 \cdot 1,14.5 \\
2 \cdot 2,12 \cdot 8\end{array}$ & $\begin{array}{l}21.5 \\
13.6\end{array}$ & $\begin{array}{l}8 \cdot 9,43.5 \\
6 \cdot 3,26 \cdot 8\end{array}$ & $\begin{array}{l}12 \cdot 1 \\
17 \cdot 5\end{array}$ & $\begin{array}{r}9.8,14.9 \\
14.8,20 \cdot 6\end{array}$ & $\begin{array}{l}0.147 \\
0.105\end{array}$ \\
\hline Choose low- or reduced-fat varieties regularly/always & $\begin{array}{l}M \\
F\end{array}$ & $\begin{array}{l}38.5 \\
44.5\end{array}$ & $\begin{array}{l}24 \cdot 5,54 \cdot 8 \\
27 \cdot 8,62 \cdot 5\end{array}$ & $\begin{array}{l}41.4 \\
34.6\end{array}$ & $\begin{array}{l}25 \cdot 0,60 \cdot 0 \\
21 \cdot 0,51 \cdot 3\end{array}$ & $\begin{array}{l}33 \cdot 7 \\
47 \cdot 4\end{array}$ & $\begin{array}{l}29.9,37.8 \\
43.8,51.0\end{array}$ & $\begin{array}{l}0.587 \\
0.266\end{array}$ \\
\hline Remove excess fat from meat regularly/always & $\begin{array}{l}M \\
F\end{array}$ & $\begin{array}{l}77.4 \S \\
94.8 \S, \|\end{array}$ & $\begin{array}{l}57 \cdot 3,89 \cdot 7 \\
85 \cdot 2,98 \cdot 3\end{array}$ & $\begin{array}{l}71.5 \S \\
61.8\end{array}$ & $\begin{array}{l}54 \cdot 2,84 \cdot 1 \\
46 \cdot 5,75 \cdot 0\end{array}$ & $\begin{array}{l}57 \cdot 3 \\
69 \cdot 2\end{array}$ & $\begin{array}{l}53 \cdot 1,61 \cdot 3 \\
65 \cdot 8,72 \cdot 5\end{array}$ & $\begin{array}{l}0.041 \\
0.018\end{array}$ \\
\hline Remove skin from chicken regularly/always & $\begin{array}{l}\mathrm{M} \\
\mathrm{F}\end{array}$ & $\begin{array}{l}72 \cdot 6 \S \\
84 \cdot 2 \S, \|\end{array}$ & $\begin{array}{l}55 \cdot 0,85 \cdot 1 \\
63 \cdot 5,94 \cdot 2\end{array}$ & $\begin{array}{l}58 \cdot 0 \\
36 \cdot 3\end{array}$ & $\begin{array}{l}40 \cdot 4,73 \cdot 8 \\
24 \cdot 3,50 \cdot 2\end{array}$ & $\begin{array}{l}41 \cdot 8 \\
56 \cdot 3\end{array}$ & $\begin{array}{l}37 \cdot 6,46 \cdot 1 \\
52 \cdot 6,59 \cdot 9\end{array}$ & $\begin{array}{r}0.001 \\
<0.001\end{array}$ \\
\hline Use vegetable oils for cooking food & $\begin{array}{l}M \\
F\end{array}$ & $\begin{array}{l}94 \cdot 6 \\
100\end{array}$ & $\begin{array}{c}75 \cdot 9,99 \cdot 0 \\
-\end{array}$ & $\begin{array}{l}97 \cdot 0 \\
92 \cdot 0\end{array}$ & $\begin{array}{l}84.0,99.5 \\
77 \cdot 0,97 \cdot 5\end{array}$ & $\begin{array}{l}90 \cdot 3 \\
90 \cdot 8\end{array}$ & $\begin{array}{l}87 \cdot 7,92 \cdot 3 \\
88 \cdot 8,92 \cdot 6\end{array}$ & $\begin{array}{l}0.457 \\
0.584\end{array}$ \\
\hline Fast food consumed more than three times per week & $\begin{array}{l}\mathrm{M} \\
\mathrm{F}\end{array}$ & $\begin{array}{r}19.2 \S \\
0.4 \S\end{array}$ & $\begin{array}{l}8 \cdot 9,36 \cdot 6 \\
0 \cdot 1,2 \cdot 8\end{array}$ & $\begin{array}{l}13.9 \\
6.5 \S, \|\end{array}$ & $\begin{array}{l}5 \cdot 6,30 \cdot 4 \\
2 \cdot 6,15 \cdot 5\end{array}$ & $\begin{array}{l}6 \cdot 7 \\
2 \cdot 4\end{array}$ & $\begin{array}{l}4.9,9 \cdot 1 \\
1 \cdot 6,3 \cdot 5\end{array}$ & $\begin{array}{l}0.014 \\
0.027\end{array}$ \\
\hline Soft drinks consumed more than three times per week & $\begin{array}{l}\mathrm{M} \\
\mathrm{F}\end{array}$ & $\begin{array}{c}30 \cdot 7 \\
7.4 \S\end{array}$ & $\begin{array}{r}18 \cdot 0,47 \cdot 4 \\
3.0,16 \cdot 9\end{array}$ & $\begin{array}{l}29 \cdot 4 \\
6 \cdot 7 \S\end{array}$ & $\begin{array}{r}17 \cdot 0,45 \cdot 8 \\
2 \cdot 8,15 \cdot 3\end{array}$ & $\begin{array}{l}29 \cdot 8 \\
17 \cdot 4\end{array}$ & $\begin{array}{l}26 \cdot 1,33 \cdot 8 \\
14 \cdot 8,20 \cdot 4\end{array}$ & $\begin{array}{l}0.991 \\
0.008\end{array}$ \\
\hline Alcohol consumption & & & & & & & & \\
\hline Any in the past 12 months & $\begin{array}{l}M \\
F\end{array}$ & $\begin{array}{l}81 \cdot 9 \S \\
47 \cdot 3 \S, \|\end{array}$ & $\begin{array}{l}70 \cdot 1,93 \cdot 7 \\
29 \cdot 2,65 \cdot 5\end{array}$ & $\begin{array}{l}85 \cdot 5 \\
70 \cdot 4 \S\end{array}$ & $\begin{array}{l}74 \cdot 8,96 \cdot 2 \\
57 \cdot 1,83 \cdot 6\end{array}$ & $\begin{array}{l}93.5 \\
87.7\end{array}$ & $\begin{array}{l}91 \cdot 8,95 \cdot 1 \\
85 \cdot 5,89 \cdot 9\end{array}$ & $\begin{array}{r}0.005 \\
<0.001\end{array}$ \\
\hline Consume alcohol more than weekly $\ddagger$ & $\begin{array}{l}\mathrm{M} \\
\mathrm{F}\end{array}$ & $\begin{array}{c}53.9 \\
9.3 \S\end{array}$ & $\begin{array}{r}36.9,70.9 \\
0,21.2\end{array}$ & $\begin{array}{l}15 \S, \| \\
10 \cdot 3 \S\end{array}$ & $\begin{array}{l}3 \cdot 1,27 \cdot 0 \\
0 \cdot 8,21 \cdot 4\end{array}$ & $\begin{array}{l}59 \cdot 8 \\
46 \cdot 7\end{array}$ & $\begin{array}{l}36 \cdot 9,70 \cdot 1 \\
42 \cdot 5,50 \cdot 9\end{array}$ & $\begin{array}{l}<0.001 \\
<0.001\end{array}$ \\
\hline
\end{tabular}

SA, South Asians; ESEA; East and South-East Asians; NZEO, New Zealand Europeans and Others; M, males; F, females.

${ }^{*} P$ value from $\chi^{2}$ test.

†Other milk includes cow's milk alternatives such as rice milk and soya milk.

†Only includes those reporting alcohol consumption.

§Percentage significantly different compared with NZEO.

IIPercentage significantly different between the Asian subgroups. 
Table 3 Mean daily nutrient intakes (age-adjusted) by gender and ethnicity ( $n$ 2995), 2008/09 Adult National Nutrition Survey, New Zealand

\begin{tabular}{|c|c|c|c|c|c|c|c|}
\hline \multirow[b]{2}{*}{ Nutrient } & \multicolumn{2}{|c|}{ SA } & \multicolumn{2}{|c|}{ ESEA } & \multicolumn{2}{|c|}{ NZEO } & \multirow[b]{2}{*}{$P$ value } \\
\hline & Mean & $95 \% \mathrm{Cl}$ & Mean & $95 \% \mathrm{Cl}$ & Mean & $95 \% \mathrm{Cl}$ & \\
\hline \multicolumn{8}{|c|}{ Energy (kJ) } \\
\hline$M$ & $8352 \dagger$ & 7280,9582 & 8677 & 7272,10353 & 9802 & 9424,10195 & 0.048 \\
\hline $\mathrm{F}$ & 5513十, & 4740,6412 & 6854 & 6070,7739 & 7236 & 7043,7434 & 0.002 \\
\hline \multicolumn{8}{|c|}{ Protein (q) } \\
\hline $\mathrm{M}$ & 76 & 64,92 & 90 & 73,110 & 93 & 89,96 & 0.137 \\
\hline $\mathrm{F}$ & 50†,‡ & 42,59 & $71 \dagger$ & 62,82 & 67 & 65,69 & 0.003 \\
\hline \multicolumn{8}{|c|}{ Protein (\%TE) } \\
\hline M & $15 \cdot 7$ & $14 \cdot 3,17 \cdot 2$ & $17.9 \dagger$ & $16 \cdot 5,19 \cdot 4$ & $16 \cdot 3$ & $15 \cdot 9,16 \cdot 6$ & 0.070 \\
\hline $\mathrm{F}$ & $15 \cdot 7 \ddagger$ & $14 \cdot 1,17 \cdot 2$ & $17.8 \dagger$ & $16 \cdot 6,19 \cdot 1$ & $16 \cdot 3$ & $15 \cdot 9,16 \cdot 7$ & 0.038 \\
\hline \multicolumn{8}{|l|}{ Fat $(g)$} \\
\hline$M$ & $65 \dagger$ † & 53,80 & 70 & 56,87 & 85 & 81,89 & 0.018 \\
\hline $\mathrm{F}$ & $47 \dagger$ & 39,57 & 56 & 48,66 & 62 & 60,65 & 0.013 \\
\hline \multicolumn{8}{|c|}{ Fat (\%TE) } \\
\hline $\mathrm{M}$ & $30 \cdot 7 \ddagger$ & $27 \cdot 7,33 \cdot 7$ & 31.3 & $28.6,33.9$ & 33.8 & $33 \cdot 0,34.5$ & 0.043 \\
\hline $\mathrm{F}$ & $33.3^{\top}$ & $30 \cdot 0,36 \cdot 7$ & 31.8 & $29.4,34.2$ & 33.9 & $33.2,34.5$ & 0.276 \\
\hline Saturate & & & & & & & \\
\hline$M$ & $21.9 \dagger$ & $17 \cdot 4,27 \cdot 6$ & $23.0 \dagger$ & $18 \cdot 2,29 \cdot 0$ & $32 \cdot 8$ & $31 \cdot 1,34 \cdot 6$ & $<0.001$ \\
\hline $\mathrm{F}$ & $16 \cdot 6 t$ & $13 \cdot 1,21 \cdot 1$ & $18 \cdot 7 \dagger$ & $15 \cdot 4,22 \cdot 6$ & 23.4 & $22 \cdot 4,24.5$ & 0.003 \\
\hline Saturate & & & & & & & \\
\hline$M$ & $10.8 \dagger$ & $9 \cdot 4,12 \cdot 3$ & $10 \cdot 6 t$ & $9.5,11.8$ & $13 \cdot 3$ & $12 \cdot 9,13 \cdot 7$ & $<0.001$ \\
\hline $\mathrm{F}$ & 12.5 & $10 \cdot 3,14.8$ & $11 \cdot 1 \dagger$ & $9.8,12.5$ & 13.2 & $12 \cdot 8,13 \cdot 5$ & 0.038 \\
\hline Polyunsa & at $(g)$ & & & & & & \\
\hline$M$ & 10.6 & $8 \cdot 3,13 \cdot 5$ & $10 \cdot 8$ & $8 \cdot 3,14 \cdot 0$ & 11.5 & $11 \cdot 0,12 \cdot 1$ & 0.728 \\
\hline $\mathrm{F}$ & 7.6 & $6 \cdot 3,9 \cdot 1$ & 8.9 & $7 \cdot 4,10.7$ & 8.7 & $8.3,9.0$ & 0.338 \\
\hline Polyunsa & at (\%TE) & & & & & & \\
\hline M & 5.2 & $4 \cdot 4,6 \cdot 1$ & $5 \cdot 1$ & $4 \cdot 5,5 \cdot 7$ & 4.7 & $4.6,4.9$ & 0.310 \\
\hline $\mathrm{F}$ & $5 . \overline{5}$ & $5 \cdot 0,6 \cdot 1$ & $5 \cdot 3$ & $4.8,5.9$ & 4.9 & $4 \cdot 8,5 \cdot 1$ & 0.086 \\
\hline Monouns & fat $(\mathrm{g})$ & & & & & & \\
\hline M & 24.1 & $19 \cdot 1,30 \cdot 4$ & $27 \cdot 9$ & $22 \cdot 2,34.9$ & 30.4 & $28 \cdot 9,32 \cdot 0$ & 0.131 \\
\hline $\mathrm{F}$ & 16.4†, & $13 \cdot 8,19 \cdot 6$ & $21 \cdot 1$ & $17 \cdot 7,25 \cdot 0$ & $22 \cdot 1$ & $21 \cdot 3,23 \cdot 0$ & 0.006 \\
\hline Monouns & fat (\%TE) & & & & & & \\
\hline $\mathrm{M}$ & 11.7 & $10 \cdot 1,13 \cdot 3$ & $12 \cdot 6$ & $11 \cdot 3,13.9$ & $12 \cdot 3$ & $11 \cdot 9,12 \cdot 7$ & 0.679 \\
\hline $\mathrm{F}$ & $12 \cdot 0$ & $10 \cdot 5,13.5$ & $12 \cdot 3$ & $11 \cdot 1,13 \cdot 4$ & $12 \cdot 3$ & $12 \cdot 0,12 \cdot 6$ & 0.941 \\
\hline Carbohy & & & & & & & \\
\hline M & 246 & 217,278 & 256 & 216,302 & 262 & 252,272 & 0.617 \\
\hline $\mathrm{F}$ & 168 & 144,195 & 201 & 180,226 & 197 & 191,203 & 0.120 \\
\hline Carbohy & TE) & & & & & & \\
\hline $\mathrm{M}$ & $50 \cdot 7 \dagger$ & $46 \cdot 8,54 \cdot 5$ & $49.9 \dagger$ & $47 \cdot 5,52 \cdot 2$ & $45 \cdot 6$ & $44.8,46 \cdot 5$ & $<0.001$ \\
\hline $\mathrm{F}$ & $51.6 \dagger$ & $48 \cdot 6,54 \cdot 6$ & $49.8 \dagger$ & $47 \cdot 6,52 \cdot 1$ & $46 \cdot 7$ & $46 \cdot 0,47 \cdot 5$ & $<0.001$ \\
\hline Choleste & & & & & & & \\
\hline $\mathrm{M}$ & 136†,‡ & 92,200 & 231 & 175,307 & 249 & 231,269 & 0.015 \\
\hline $\mathrm{F}$ & 121 & 83,177 & 203 & 164,251 & 173 & 164,183 & 0.060 \\
\hline Dietary $f$ & & & & & & & \\
\hline$M$ & $19 \cdot 6$ & $16 \cdot 9,22 \cdot 7$ & $19 \cdot 6$ & $16 \cdot 1,23 \cdot 7$ & $20 \cdot 4$ & $19 \cdot 5,21 \cdot 3$ & 0.820 \\
\hline $\mathrm{F}$ & 14.8 & $12 \cdot 6,17.5$ & $17 \cdot 3$ & $15 \cdot 1,19 \cdot 7$ & $16 \cdot 3$ & $15 \cdot 7,16.9$ & 0.381 \\
\hline Total sug & & & & & & & \\
\hline M & $77 \dagger$ & 61,96 & 78 & 54,113 & 110 & 105,116 & 0.003 \\
\hline $\mathrm{F}$ & 68 & 59,78 & 75 & 62,91 & 91 & 87,95 & 0.003 \\
\hline Vitamin & & & & & & & \\
\hline$M$ & $592 \dagger$ & 484,726 & $454 \dagger$ & 323,638 & 748 & 702,796 & 0.004 \\
\hline $\mathrm{F}$ & 523 & 384,711 & 527 & 418,666 & 632 & 600,665 & 0.180 \\
\hline$\beta$-Carote & & & & & & & \\
\hline $\mathrm{M}$ & 1808 & 1408,2322 & 1398 & 937,2086 & 1761 & 1610,1927 & 0.531 \\
\hline $\mathrm{F}$ & 1567 & 944,2602 & 1691 & 1235,2315 & 1708 & 1571,1857 & 0.947 \\
\hline Retinol ( & & & & & & & \\
\hline$M$ & $198 \dagger$ & 149,262 & $144 \dagger$ & 96,216 & 332 & 306,360 & $<0.001$ \\
\hline $\mathrm{F}$ & $164 \dagger$ † & 130,208 & $156 \dagger$ & 120,204 & 235 & 221,250 & $<0.001$ \\
\hline Thiamin & & & & & & & \\
\hline M & $1 \cdot 1 \dagger$ & $0.9,1.3$ & $1 \cdot 1 \dagger$ & $0.9,1.4$ & 1.5 & $1.4,1.5$ & 0.004 \\
\hline $\mathrm{F}$ & 0.9 & $0.7,1 \cdot 1$ & $0.8 \dagger$ & $0.7,0.9$ & 1.0 & $1 \cdot 0,1 \cdot 1$ & 0.007 \\
\hline Riboflavi & & & & & & & \\
\hline$M$ & $1.5 \dagger$ & $1 \cdot 3,1 \cdot 8$ & $1.6 \dagger$ & $1 \cdot 3,2 \cdot 0$ & $2 \cdot 0$ & $1 \cdot 9,2 \cdot 1$ & 0.001 \\
\hline $\mathrm{F}$ & 1.4 & $1 \cdot 2,1 \cdot 7$ & 1.2 & $1.0,1.5$ & 1.5 & $1.5,1.6$ & 0.064 \\
\hline Niacin ec & $\mathrm{s}(\mathrm{mg})$ & & & & & & \\
\hline $\mathrm{M}$ & $14.2 \dagger$ & $11 \cdot 4,17 \cdot 6$ & $16 \cdot 6$ & $13 \cdot 4,20 \cdot 6$ & $19 \cdot 3$ & $18 \cdot 4,20 \cdot 2$ & 0.015 \\
\hline $\mathrm{F}$ & 9.7†,‡ & $8.0,11.6$ & $13 \cdot 0$ & $11.0,15 \cdot 4$ & 13.5 & $12 \cdot 9,14 \cdot 1$ & 0.003 \\
\hline Vitamin & & & & & & & \\
\hline $\mathrm{M}$ & $1.4 \dagger$ & $1 \cdot 2,1 \cdot 7$ & 1.7 & $1 \cdot 3,2 \cdot 2$ & 1.8 & $1 \cdot 7,2 \cdot 0$ & 0.036 \\
\hline $\mathrm{F}$ & 1.2 & $1.0,1.4$ & 1.4 & $1.1,1.6$ & 1.4 & $1.3,1.4$ & 0.263 \\
\hline
\end{tabular}


Table 3 Continued

\begin{tabular}{|c|c|c|c|c|c|c|c|}
\hline \multirow[b]{2}{*}{ Nutrient } & \multicolumn{2}{|c|}{ SA } & \multicolumn{2}{|c|}{ ESEA } & \multicolumn{2}{|c|}{ NZEO } & \multirow[b]{2}{*}{$P$ value } \\
\hline & Mean & $95 \% \mathrm{Cl}$ & Mean & $95 \% \mathrm{Cl}$ & Mean & $95 \% \mathrm{Cl}$ & \\
\hline \multicolumn{8}{|c|}{ Vitamin $B_{1}(m g)$} \\
\hline$M$ & $1.9 \dagger$ & $1.4,2.6$ & $2 \cdot 7$ & $1.9,4.0$ & 3.7 & $3.5,4.0$ & $<0.001$ \\
\hline $\mathrm{F}$ & $1.6 \dagger$, & $1 \cdot 2,2 \cdot 1$ & $2 \cdot 7$ & $2 \cdot 1,3.5$ & $2 \cdot 5$ & $2 \cdot 4,2 \cdot 7$ & 0.003 \\
\hline \multicolumn{8}{|c|}{ Vitamin C (mg) } \\
\hline M & 71 & 55,92 & 77 & 50,118 & 68 & 62,75 & 0.851 \\
\hline $\mathrm{F}$ & 61 & 45,84 & 89 & 64,123 & 71 & 66,76 & 0.276 \\
\hline \multicolumn{8}{|c|}{ Vitamin E (mg) } \\
\hline $\mathrm{M}$ & 9.2 & $7.4,11.5$ & $9 \cdot 1$ & $7 \cdot 0,12 \cdot 0$ & $10 \cdot 3$ & $9 \cdot 8,10 \cdot 9$ & 0.456 \\
\hline $\mathrm{F}$ & 7.4 & $6 \cdot 4,8 \cdot 6$ & 8.5 & $7 \cdot 2,10 \cdot 1$ & 8.4 & $8 \cdot 0,8 \cdot 7$ & 0.282 \\
\hline \multicolumn{8}{|l|}{$\mathrm{Ca}(\mathrm{mg})$} \\
\hline$M$ & $598 \dagger$ & 506,707 & $554 \dagger$ & 420,731 & 856 & 816,898 & $<0.001$ \\
\hline F & 605 & 498,736 & $501 \dagger$ & 407,618 & 695 & 668,724 & 0.006 \\
\hline \multicolumn{8}{|l|}{$\mathrm{Fe}(\mathrm{mg})$} \\
\hline $\mathrm{M}^{\prime}$ & 11.1 & $9.5,12 \cdot 9$ & 11.5 & $9 \cdot 2,14 \cdot 4$ & $12 \cdot 3$ & $11 \cdot 8,12 \cdot 8$ & 0.398 \\
\hline $\mathrm{F}$ & 7.8 & $6 \cdot 7,9 \cdot 2$ & 9.7 & $8.5,11.0$ & $9 \cdot 2$ & $8.9,9.5$ & 0.124 \\
\hline \multicolumn{8}{|l|}{$\mathrm{K}(\mathrm{mg})$} \\
\hline M & 2704†,‡ & 2305,3171 & 2846 & 2302,3520 & 3243 & 3123,3368 & 0.056 \\
\hline $\mathrm{F}$ & $2213+$ & 1940,2525 & 2429 & 2049,2880 & 2620 & 2538,2705 & 0.043 \\
\hline \multicolumn{8}{|l|}{ Se $(m g)$} \\
\hline$M$ & $39.0 \dagger$ & $31 \cdot 6,48 \cdot 2$ & 48.9 & $36 \cdot 3,65 \cdot 9$ & 53.7 & $50 \cdot 6,56 \cdot 9$ & 0.019 \\
\hline F & $26.5 \dagger, \ddagger$ & $20 \cdot 6,34.0$ & $47.4 \dagger$ & $40 \cdot 3,55 \cdot 8$ & 38.9 & $37 \cdot 2,40 \cdot 7$ & $<0.001$ \\
\hline \multicolumn{8}{|l|}{$\mathrm{Zn}(\mathrm{mq})$} \\
\hline$M$ & $11 \cdot 1$ & $9 \cdot 1,13 \cdot 6$ & 11.9 & $9 \cdot 7,14 \cdot 6$ & 11.4 & $11 \cdot 0,11.9$ & 0.900 \\
\hline $\mathrm{F}$ & 6.5†, & $5 \cdot 5,7 \cdot 7$ & $9.7 \dagger$ & $8.5,11.0$ & 8.2 & $7.9,8.5$ & 0.018 \\
\hline
\end{tabular}

SA, South Asians; ESEA; East and South-East Asians; NZEO, New Zealand Europeans and Others; M, males; F, females; \%TE, percentage of total energy; $R E$, retinol equivalents.

Geometric mean reported for all nutrients apart from \%TE.

${ }^{*} P$ value for the association between nutrient intake and ethnicity.

†Mean value significantly different compared with NZEO.

$\ddagger$ Mean significantly different between Asian subgroups.

categorized with Fe-deficiency anaemia (4.5\%) compared with NZEO $(P<0 \cdot 05)$. ESEA females had lower Hb levels compared with NZEO females $(P<0 \cdot 05$; Table 4$)$.

Mean RBC folate concentrations were similar among all three ethnic groups for males and females (males $P=0.355$; females $P=0 \cdot 916$ ). The percentage of SA males with low RBC folate concentration was higher than NZEO $(P<0.005)$ and ESEA males $(P<0.005$; Table 4$)$.

Among females the prevalence of diagnosed diabetes was significantly higher $(P<0.001)$ among SA $(20.5 \%)$ compared with ESEA $(0 \cdot 3 \%)$ and NZEO females (2.8\%). None of the ESEA males reported having diabetes and SA males reported a significantly higher prevalence of diagnosed diabetes compared with NZEO males. Mean HbA1c level among SA males (6.1\%) was higher than that among ESEA (5.6\%; $P<0.05)$ and NZEO $(5.5 \% ; P<0.05)$ males. Twenty-seven per cent of SA males ( $>$ NZEO; $P<0 \cdot 010$ ) and about $24 \%$ of SA females had HbA1c levels equal to or above $6.5 \%$ (Table 4 ).

Mean diastolic and systolic pressure was similar among males and females of the three ethnic groups.

\section{Body size}

Figure 1(a) and (b) presents the age-adjusted prevalence of overweight and obesity among males and females by ethnicity using standard cut-offs and ethnic-specific cut-offs. Using standard cut-offs (Fig. 1(a)), SA males and females were more likely to be overweight compared with ESEA
$(P<0.05)$ but were similar to NZEO. ESEA males and females had lower prevalence of being overweight and obese compared with NZEO $(P<0 \cdot 05)$.

Using ethnic-specific cut-offs increased the prevalence of obesity among the Asian subgroups (Fig. 1(b)). Higher percentage of SA males (66\%) was obese when compared with ESEA $(37 \% ; P<0.05)$ and NZEO $(27 \% ; P<0.001)$ males. Among females $56 \%$ of SA were obese, which was higher than both ESEA ( $22 \% ; P=0.007)$ and NZEO (25\%; $P=0 \cdot 003)$.

\section{Discussion}

The results presented in the current paper are unique, and report nutrient intakes, dietary habits and health outcomes from national survey data for South Asians and East/South-East Asians separately. Such analysis has enabled comparisons between the two Asian subgroups, thereby providing new knowledge on similarities and differences in dietary intakes and health outcomes. Some significant differences according to duration of residence have also been reported, particularly for SA males.

A different dietary profile was evident for the Asian subgroups with higher percentage of energy from carbohydrate than NZEO due to their predominantly carbohydrate-based diets. A unique aspect of the SA subgroup was that, in contrast to ESEA, SA reported consuming lower amounts 
Table 4 Blood indices and prevalence of diagnosed diabetes and blood pressure (age-adjusted) by gender and ethnicity, 2008/09 Adult National Nutrition Survey, New Zealand

\begin{tabular}{|c|c|c|c|c|c|c|c|}
\hline \multirow[b]{2}{*}{ Blood index } & \multicolumn{2}{|c|}{$\begin{array}{c}\mathrm{SA} \\
(\mathrm{M}, n 39 ; \mathrm{F}, n 40)\end{array}$} & \multicolumn{2}{|c|}{$\begin{array}{c}\text { ESEA } \\
(\mathrm{M}, n 28 ; \mathrm{F}, n \text { 55) }\end{array}$} & \multicolumn{2}{|c|}{$\begin{array}{c}\text { NZEO } \\
(\mathrm{M}, n \text { 908; F, } n \text { 1169) }\end{array}$} & \multirow[b]{2}{*}{$P$ value } \\
\hline & Mean & $95 \% \mathrm{Cl}$ & Mean & $95 \% \mathrm{Cl}$ & Mean & $95 \% \mathrm{Cl}$ & \\
\hline \multicolumn{8}{|c|}{ Total cholesterol (mmol/l) } \\
\hline M & 5.4 & $5 \cdot 0,5 \cdot 7$ & $5 \cdot 3$ & $4 \cdot 9,5 \cdot 7$ & $5 \cdot 1$ & $4 \cdot 9,5 \cdot 2$ & $0 \cdot 181$ \\
\hline $\mathrm{F}$ & $5 \cdot 2$ & $4 \cdot 8,5 \cdot 6$ & $5 \cdot 0$ & $4 \cdot 7,5 \cdot 3$ & $5 \cdot 2$ & $5 \cdot 1,5 \cdot 3$ & 0.537 \\
\hline \multicolumn{8}{|c|}{ HDL-cholesterol (mmol/l) } \\
\hline M & $1 \cdot 21$ & $1.09,1.32$ & 1.33 & $1.22,1.44$ & 1.24 & $1 \cdot 21,1 \cdot 26$ & 0.203 \\
\hline $\mathrm{F}$ & 1.39 & $1.28,1.51$ & 1.57 & $1.45,1.70$ & 1.53 & $1.50,1.56$ & 0.074 \\
\hline \multicolumn{8}{|c|}{ Total cholesterol:HDL-cholesterol } \\
\hline M & 4.66 & $4 \cdot 21,5 \cdot 10$ & $4 \cdot 14$ & $3.69,4.59$ & $4 \cdot 30$ & $4.18,4.59$ & 0.234 \\
\hline $\mathrm{F}$ & 3.86 & $3.45,4.27$ & $3 \cdot 31$ & $3.07,3.59$ & 3.57 & $3.49,3.66$ & 0.094 \\
\hline \multicolumn{8}{|l|}{ Ferritin $(\mu \mathrm{g} / \mathrm{l})$} \\
\hline M & $124^{\star}$ & 76,171 & 201 & 152,250 & 177 & 160,193 & 0.059 \\
\hline $\mathrm{F}$ & $60^{*}, \dagger$ & 48,71 & 81 & 59,102 & 79 & 74,83 & 0.010 \\
\hline \multicolumn{8}{|c|}{$\%$ Low Fe stores $(<12 \mu \mathrm{g} / \mathrm{l})$} \\
\hline M & $17 \cdot 0^{*}$ & $1 \cdot 1,32 \cdot 2$ & $0.0 \ddagger$ & - & 0.7 & $0.0,1.3$ & $<0.001$ \\
\hline $\mathrm{F}$ & $16 \cdot 3$ & $0.0,33.6$ & $12 \cdot 3$ & $1 \cdot 1,23.5$ & 8.2 & $5.8,10.5$ & 0.396 \\
\hline \multicolumn{8}{|l|}{$\mathrm{Hb}(\mathrm{g} / \mathrm{l})$} \\
\hline M & 145 & 140,151 & 148 & 145,152 & 149 & 148,150 & 0.301 \\
\hline $\mathrm{F}$ & 128 & 122,134 & $129^{*}$ & 125,132 & 133 & 132,134 & 0.017 \\
\hline \multicolumn{8}{|l|}{$\%$ Low $\mathrm{Hb}$} \\
\hline $\mathrm{M}$ & $14 \cdot 1^{*}$ & $0,28 \cdot 1$ & $0.9^{*}, \dagger$ & $0 \cdot 0,2 \cdot 6$ & $5 \cdot 3$ & $3 \cdot 5,7 \cdot 0$ & 0.036 \\
\hline $\mathrm{F}$ & $25 \cdot 1^{*}$ & $6 \cdot 7,43 \cdot 6$ & $10 \cdot 9$ & $0.0,22.9$ & $7 \cdot 3$ & $5 \cdot 0,9 \cdot 6$ & 0.031 \\
\hline \multicolumn{8}{|c|}{$\%$ Fe deficiency } \\
\hline M & $12 \cdot 7^{\star}$ & $0.0,26 \cdot 6$ & $0.0 \ddagger$ & - & 0.6 & $0.0,1.2$ & $<0.001$ \\
\hline $\mathrm{F}$ & $13 \cdot 8$ & $0.0,29 \cdot 0$ & $9 \cdot 1$ & $3 \cdot 5,19 \cdot 1$ & $6 \cdot 7$ & $4 \cdot 6,8 \cdot 8$ & 0.464 \\
\hline \multicolumn{8}{|c|}{$\%$ Fe deficiency with anaemia } \\
\hline M & $4 \cdot 5^{\star}$ & $0.0,11 \cdot 6$ & $0.0 \ddagger$ & - & 0.2 & $0.0,4 \cdot 0$ & $<0.001$ \\
\hline $\mathrm{F}$ & 4.6 & $0.0,13 \cdot 1$ & $7 \cdot 1$ & $0 \cdot 0,16 \cdot 2$ & 3.4 & $1.9,4.9$ & 0.597 \\
\hline \multicolumn{8}{|c|}{ RBC folate $(\mathrm{nmol} / \mathrm{l})$} \\
\hline M & 849 & 710,987 & 876 & 701,1051 & 943 & 902,985 & 0.355 \\
\hline $\mathrm{F}$ & 923 & 724,1121 & 891 & 775,1005 & 915 & 878,953 & 0.916 \\
\hline \multicolumn{8}{|c|}{$\%$ Low RBC folate $(<317 \mathrm{nmol} / \mathrm{l})$} \\
\hline M & $10 \cdot 4^{*}, \dagger$ & $0 \cdot 0,22 \cdot 7$ & 0.5 & $0.0,1 \cdot 6$ & $1 \cdot 2$ & $0 \cdot 1,2 \cdot 3$ & 0.013 \\
\hline $\mathrm{F}$ & 6.8 & $0.0,16 \cdot 1$ & $0.0 \ddagger$ & - & $2 \cdot 8$ & $1 \cdot 3,4.4$ & 0.270 \\
\hline \multicolumn{8}{|c|}{ Serum folate $(\mathrm{nmol} / \mathrm{l})$} \\
\hline M & 21.9 & $17 \cdot 0,26 \cdot 9$ & 28.4 & $21 \cdot 4,35.5$ & 27.4 & $25 \cdot 5,29 \cdot 3$ & 0.108 \\
\hline $\mathrm{F}$ & 33.0 & $22 \cdot 6,43 \cdot 5$ & 34.8 & $28 \cdot 8,40 \cdot 8$ & $31 \cdot 7$ & $29 \cdot 6,33 \cdot 8$ & 0.606 \\
\hline$\%$ Low seru & $<6.8 \mathrm{mmol}$ & & & & & & \\
\hline M & 4.8 & $0,13 \cdot 3$ & $0.0 \ddagger$ & - & 1.9 & $0.7,3.3$ & 0.358 \\
\hline $\mathrm{F}$ & 0.7 & $0.0,2.3$ & 1.0 & $0.0,2.5$ & 1.6 & $0.7,3 \cdot 2$ & 0.657 \\
\hline$\%$ Diagnose & & & & & & & \\
\hline M & $22 \cdot 8^{\star}$ & $9 \cdot 0,36 \cdot 5$ & $0.0 \ddagger$ & - & 5.4 & $3 \cdot 8,7 \cdot 1$ & $<0.001$ \\
\hline $\mathrm{F}$ & $20 \cdot 5^{\star}, \dagger$ & $5 \cdot 7,35 \cdot 2$ & $0.3^{*}$ & $0,1 \cdot 1$ & $2 \cdot 8$ & $1.9,3.7$ & $<0.001$ \\
\hline $\mathrm{HbA1c}(\%)$ & & & & & & & \\
\hline $\mathrm{M}$ & $6 \cdot 09^{*}, \dagger$ & $5 \cdot 80,6 \cdot 39$ & $5 \cdot 62$ & $5 \cdot 49,5 \cdot 77$ & $5 \cdot 52$ & $5 \cdot 47,5.52$ & $<0.001$ \\
\hline $\mathrm{F}$ & 5.83 & $5.46,6.19$ & $5 \cdot 46^{\star}, \dagger$ & $5.36,5.57$ & 5.46 & $5.42,5.49$ & 0.056 \\
\hline$\%$ High $\mathrm{HbA}$ & $\%)$ & & & & & & \\
\hline$M^{\circ}$ & $27 \cdot 7^{*}$ & $13 \cdot 4,42 \cdot 0$ & $0.0 \ddagger$ & - & 5.4 & $3 \cdot 8,7 \cdot 0$ & $<0.001$ \\
\hline $\mathrm{F}$ & $24 \cdot 3^{\star}$ & $3.5,45 \cdot 0$ & $2 \cdot 7^{\circ}$ & $0.0,6 \cdot 2$ & $3 \cdot 2$ & $2 \cdot 1,4 \cdot 1$ & 0.002 \\
\hline Systolic bloc & re $(\mathrm{mmHg})$ & & & & & & \\
\hline M & 125.9 & $115 \cdot 5,136 \cdot 3$ & $127 \cdot 3$ & $121 \cdot 7,133 \cdot 0$ & $125 \cdot 2$ & $123 \cdot 0,127 \cdot 4$ & 0.772 \\
\hline $\mathrm{F}$ & $110 \cdot 8$ & $103 \cdot 2,118.4$ & 114.8 & $110 \cdot 6,119.0$ & $116 \cdot 8$ & $114.7,119.0$ & 0.248 \\
\hline Diastolic blo & ure $(\mathrm{mmHg}$ & & & & & & \\
\hline M & 74.7 & $69 \cdot 1,80 \cdot 2$ & 73.0 & $68 \cdot 8,77 \cdot 2$ & $71 \cdot 2$ & $69 \cdot 7,72 \cdot 6$ & 0.369 \\
\hline $\mathrm{F}$ & $67 \cdot 1$ & $61 \cdot 9,72 \cdot 2$ & 68.7 & $66 \cdot 0,71 \cdot 3$ & $70 \cdot \overline{3}$ & $68.9,71.6$ & 0.313 \\
\hline
\end{tabular}

SA, South Asians; ESEA; East and South-East Asians; NZEO, New Zealand Europeans and Others; M, males; F, females; RBC, red blood cell; HbA1c, glycated $\mathrm{Hb}$.

*Mean or percentage significantly different compared with NZEO.

†Mean or percentage significantly different between Asian subgroups.

$\ddagger$ Analyses not adjusted for age due to lack of observations.

of meat, poultry and processed meats than NZEO. Consequently, SA also had lower intakes of fat, protein (among females), saturated fat and cholesterol (among males). Vitamin and mineral intakes, namely retinol, niacin, vitamin $\mathrm{B}_{12}$ and $\mathrm{Zn}$, were also lower among $\mathrm{SA}$ females.

Given the high percentage of SA reporting they never eat red meat or chicken (Table 2), it is not surprising that 

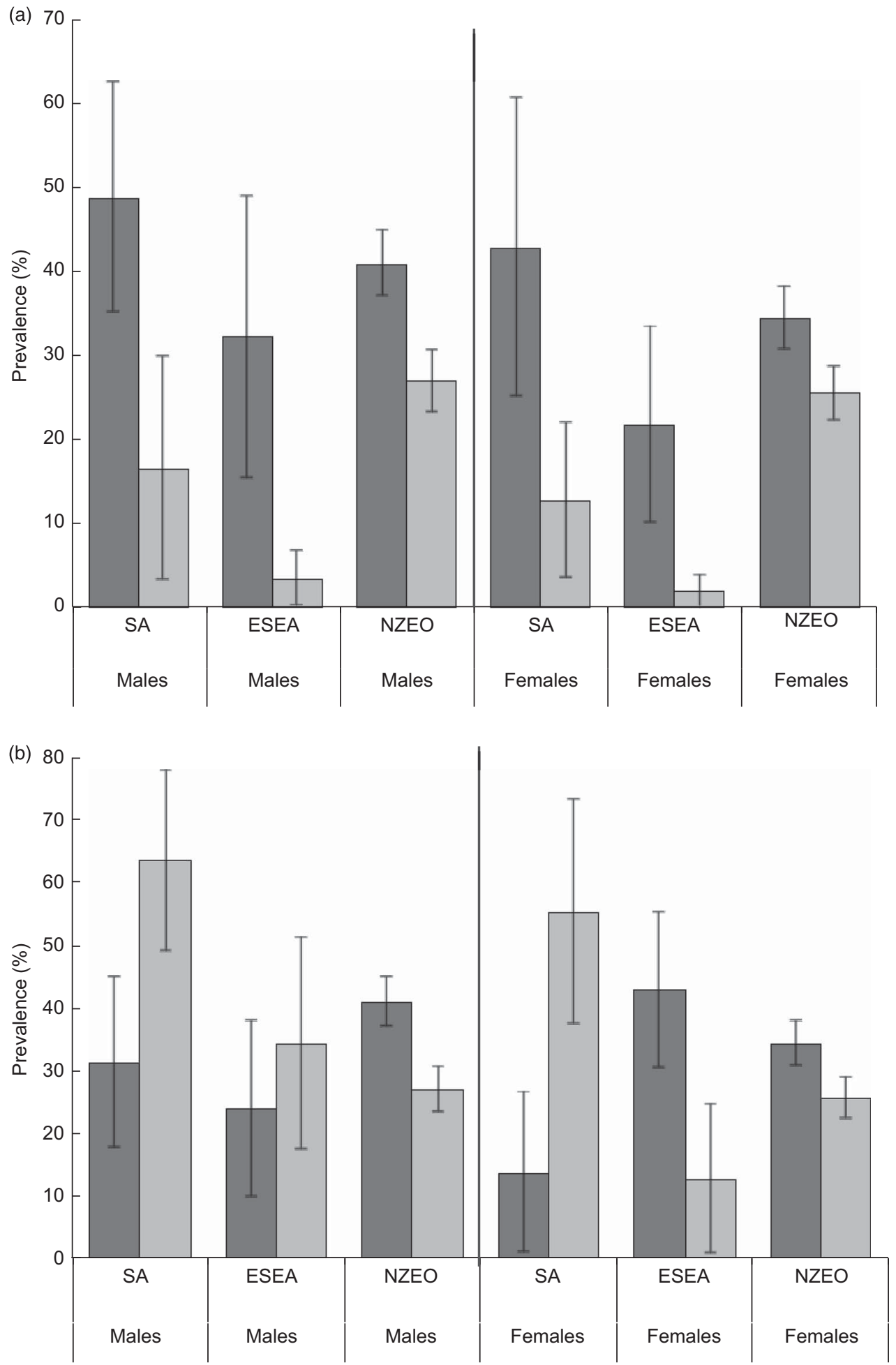

Fig. 1 Percentage of (age-adjusted) overweight $(\square$ ) and obesity $(\square)$ by gender and ethnicity (SA, South Asians; ESEA; East and SouthEast Asians; NZEO, New Zealand Europeans and Others), using (a) standard cut-offs and (b) ethnic-specific cut-offs, among adults aged 15 years and older ( $n$ 2995), 2008/09 Adult National Nutrition Survey, New Zealand. Values are means with their $95 \%$ confidence intervals represented by vertical bars

biochemical indices of Fe status were poorer for SA males and females (although not statistically significant in females). Dietary Fe intake was not significantly lower in SA but it is likely to be coming from non-haem sources and therefore is not as bioavailable. Having an 'Asian dietary pattern' has been found to be a risk factor for suboptimal Fe status in women of childbearing age ${ }^{(40)}$. However, the results of our study seem to imply that 
suboptimal Fe status is more likely to be associated with SA dietary patterns only and not ESEA. These results highlight the importance of accounting for variations in dietary patterns within Asian subgroups to understand their dietrelated disease risk factors.

Consistent with other New Zealand ${ }^{(4)}$ and Australian research $^{(41)}$, Ca intakes were lower among SA males and ESEA males and females compared with NZEO. Further examination of the food groups reported in the $24 \mathrm{~h}$ diet recall (data not shown) reveals that SA and ESEA males and females were less likely to report cheese and ESEA were less likely to report milk and other dairy products compared with NZEO. This may partly explain the lower $\mathrm{Ca}$ intakes of the Asian subgroups.

No ethnic differences were observed for serum cholesterol levels among males. However, in the NZ Health Survey (NZHS), SA males had twice the risk for hypercholesterolaemia $^{(42)}$. On further examination of the data in the current study, it was found that the percentage of SA males on medication was double that of NZEO; however as the number of SA was small ( $n 7$; data not shown), it was not feasible to analyse excluding those on medication.

Consistent with the results of the NZHS ${ }^{(42)}$, lower proportions of the Asian subgroups met the recommendation for servings of fruits and vegetables. However, caution should be exercised in interpreting these results from the dietary habits questionnaire because this recommendation is based on serving sizes, the concept of which may vary within Asian subgroups as well as between Asians and Europeans. Higher percentages of SA females and SA males had poor RBC folate status and poor serum folate status, respectively, which provides some evidence for lower fruit and vegetable intakes in the SA subgroup. Both serum folate and RBC folate concentrations were reported as the former is indicative of recent folate intake and the latter reflects long-term folate status ${ }^{(43)}$.

In contrast to females, SA males were more likely to consume fast food 'more than three times per week' than NZEO. The reason for this observed gender difference is unclear and warrants further investigation, especially as SA males are at high risk for diet-related chronic diseases ${ }^{(43)}$.

Although some of the differences observed did not reach statistical significance due to small sample size, the magnitude of the prevalence of diagnosed diabetes among SA in comparison with ESEA and NZEO cannot be ignored. Results from the NZHS also corroborate these findings as the prevalence of diabetes among SA (no gender differences were reported) was $12 \%{ }^{(42)}$. Prevalence of diabetes in a national study in the USA was $17 \cdot 4 \%$ among Asian Indians ${ }^{(13)}$. These findings are consistent with those observed in India, which leads the world in the number of diabetic cases ${ }^{(44)}$. Although the primary driver of the diabetic epidemic in India is nutrition transition ${ }^{(45)}$, there is some evidence that genetic predisposition also plays a role ${ }^{(46)}$.

Using ethnic-specific cut-offs, the prevalence of obesity among the SA males and females was much higher than among NZEO and ESEA females $(P<0 \cdot 01)$. A linear relationship was observed between duration of residence and the prevalence of overweight and obesity (combined) in the $\mathrm{NZHS}^{(42)}$. However, this was not observed in the current study; our findings were similar to those observed among migrants from India and South-East Asia in a US study ${ }^{(47)}$ and in a small sample of SA women in Australia ${ }^{(48)}$.

A strength of the present study is the inclusion of biochemical indices, dietary habits, nutrient intakes and anthropometry which together provide an overview of nutritional status. A limitation of the study is the small sample size in the Asian subgroups, especially for biochemical indices. Furthermore, adjustments for potential confounders such as age were not possible for some analyses. Similarly, a lack of SA and ESEA participants in the repeat $24 \mathrm{~h}$ recall also limited the study from reporting usual nutrient intakes and hence calculating the probability of inadequate nutrient intakes. A single $24 \mathrm{~h}$ diet recall does not capture usual intake; however, 'means' are adequate for making comparisons between subgroups of the population ${ }^{(50)}$ but may provide unreliable results with smaller numbers. As with other types of self-reported dietary assessment, under-reporting may be an issue ${ }^{(49)}$. As it was not possible to discern under-reporters from underconsumers all participants were included in the analyses. Studies have highlighted the difficulties in assessing individual consumption of foods among collective societies such as 'Asian', (50) linked to, for example, the practice of shared eating (i.e. from one dish), understanding of food terms and concepts, inadequate information on ethnic dishes and recipes, and culture-specific foods and portion sizes ${ }^{(50,51)}$.

A recent review has highlighted the need to develop and employ specific methodology to assess dietary intake among immigrant populations ${ }^{(52)}$. An NZ study on SA women has reported a significant decrease in underreporting, especially for use of plant oils and dairy, by using multiple methods for dietary data collection ${ }^{(53)}$. In preparation for the 2008/09 NZANS the $24 \mathrm{~h}$ recall probes were revised to capture ethnic-specific dishes. However, of note was the lower energy intake of SA females compared with the other ethnic groups, similar to that reported in the USA for females of an SA subgroup ${ }^{(54)}$. As energy intake was similar for SA males compared with NZEO males and ESEA females compared with NZEO females, perhaps the $24 \mathrm{~h}$ recall probes were adequate. Possible reasons for low energy intake among SA females may include under-reporting due to the effect of social desirability and language barriers. A lower level of physical activity among SA females compared with European females has been documented elsewhere ${ }^{(55)}$ and this may also explain decreased energy intakes.

The results of the current study indicate that dietary habits, nutrient intakes, blood indices and body size differ significantly between Asian subgroups and emphasize the need to acknowledge this heterogeneity in future dietand disease-related research. Investigating associations 
between health outcomes and dietary intakes according to ethnicity was beyond the scope of the current research.

\section{Conclusions}

The current study is unique in reporting dietary data and health outcomes for two Asian subgroups separately, thereby addressing in part the heterogeneity in the disease risk profile of 'Asian' people. It also provides some evidence for changes in dietary intakes according to duration of residence, especially for SA males. Investigating diet-related disease risk levels for Asian ethnic groups is imperative and hence needs to be addressed in future studies. The authors recommend that future national surveys include an adequate sample of the Asian subgroups to be able to draw comparisons and make recommendations for the public health status of these groups.

\section{Acknowledgements}

Financial support: The New Zealand Ministry of Health funded the 2008/09 New Zealand Adult Nutrition Survey. The New Zealand Crown is the owner of the copyright for the survey data. The funder had no role in the design, analysis or writing of this article. The results presented in this paper are the work of the authors. Conflict of interest: None. Authorship: S.M.P. developed the concept, developed the Asian subgroup categorization and contributed significantly to writing the manuscript. C.S. developed the analysis plan, conducted the data analysis and interpretation, wrote the Methods section and contributed towards editing the other sections of the manuscript. W.R.P. contributed to collection of data, data interpretation and editing of the manuscript. Ethics of buman subject participation: The study was conducted according to the guidelines laid down in the Declaration of Helsinki and all procedures involving human participants were approved by the Multi-Region Ethics Committee. Written informed consent was obtained from all participants.

\section{References}

1. Statistics New Zealand (2010) National Ethnic Population Projections: 2006 (base)-2026 Update. Wellington: Statistics New Zealand; available at http://www.stats.govt.nz/browse_for_stats/population/estimates_and_projections/National EthnicPopulationProjections_HOTP2006-26.aspx

2. Wahlqvist ML (2002) Asian migration to Australia: food and health consequences. Asia Pac J Clin Nutr 11, Suppl. 3, S562-S568.

3. Yang EJ, Chung HK, Kim WY et al. (2007) Chronic diseases and dietary changes in relation to Korean Americans' length of residence in the United States. J Am Diet Assoc 107, 942-950.

4. Metcalf AP, Scragg R, Schaaf D et al. (2008) Dietary intakes of European, Māori, Pacific and Asian adults living in Auckland: the Diabetes, Heart and Health Study. Aust NZJ Public Health 32, 454-460.
5. Gilbert PA \& Khokar S (2008) Changing dietary habits of ethnic groups in Europe and implications for health. Nutr Rev 66, 203-215.

6. Mente A, de Koning L, Shannon HS et al. (2009) A systematic review of the evidence supporting a causal link between dietary factors and coronary heart disease. Arch Intern Med 169, 659-669.

7. Holmboe-Ottesen G \& Wandel M (2012) Changes in dietary habits after migration and consequences for health: a focus on South Asians in Europe. J Food Nutr 56, 188-191.

8. Kolt G, Schofield GM, Rush EC et al. (2007) Body fatness, physical activity and nutritional behaviours in Asian Indian immigrants to New Zealand. Asia Pac J Clin Nutr 16, 663-670.

9. Kuppuswamy VC \& Gupta S (2005) Excess coronary heart disease in South Asians in the United Kingdom. BMJ 330, $1223-1224$.

10. Ministry of Health (2006) Asian Health Chart Book. Wellington: Ministry of Health.

11. Barnett AH, Dixon AN, Bellary S et al. (2006) Type 2 diabetes and cardiovascular risk in the UK South Asian community. Diabetologia 49, 2234-2246.

12. Misra R, Patel T, Kotha P et al. (2010) Prevalence of diabetes, metabolic syndrome and cardiovascular risk factors in US Asian Indians: results from a national study. J Diabetes Complications 24, 145-153.

13. Kanya AM, Wassel Cl, Mathur D et al. (2010) Prevalence and correlates of diabetes in South Asian Indians in the United States: findings from the metabolic syndrome and atherosclerosis in South Asians living in America study and the multi-ethnic study of atherosclerosis. Metab Syndr Relat Disord 8, 157-162.

14. Garduno-Diaz SD \& Khokhar S (2012) Prevalence, risk factors and complications associated with type 2 diabetes in migrant South Asians. Diabetes Metab Res Rev 28, 6-24.

15. Hanley AJG, Choi BCK \& Holowaty EJ (1995) Cancer mortality among Chinese migrants: a review. Int J Epidemiol 24, 255-264.

16. Virk R, Gill S, Yoshida E et al. (2010) Racial differences in the incidence of colorectal cancer. Can J Gasteroenterol 24 , $47-51$.

17. Harding S (2003) Mortality of migrants from the Indian subcontinent to England and Wales: effect of duration of residence. Epidemiology 14, 287-292.

18. Creatore MI, Moineddin R, Booth G et al. (2010) Age and sex related prevalence of diabetes mellitus among immigrants to Ontario, Canada. CMAJ 182, 781-789.

19. Dekker LH, Snijder MB, Beukers MH et al. (2011) A prospective cohort study of dietary patterns of non-western migrants in the Netherlands in relation to risk factors for cardiovascular diseases: HELIUS-Dietary Patterns. BMC Public Health 11, 441.

20. Joshi P, Islam S, Pais P et al. (2007) Risk factors for early myocardial infarction in South Asians compared with individuals in other countries. JAMA 297, 286-294.

21. Eapen D, Karla GL, Merchant N et al. (2009) Metabolic syndrome and cardiovascular disease in South Asians. Vasc Health Risk Manag 5, 731-743.

22. Tam CY, Martin LJ, Hislop G et al. (2010) Risk factors for breast cancer in postmenopausal Caucasian and ChineseCanadian women. Breast Cancer Res 12, R2.

23. Huang B, Rodriguez BL, Burchfiel CM et al. (1996) Acculturation and prevalence of diabetes among JapaneseAmerican men in Hawaii. Am J Epidemiol 144, 674-681.

24. Neuhouser ML, Thomson B, Coronado G et al. (2004) Higher fat intake and lower fruit and vegetables intakes are associated with greater acculturation among Mexicans living in Washington State. J Am Diet Assoc 104, 51-57.

25. Satia JA (2010) Dietary acculturation and the nutrition transition: an overview. Appl Physiol Nutr Metab 35, 219-223. 
26. Smith NR, Kelly YJ \& Nazroo JY (2012) The effects of acculturation on obesity rates in ethnic minorities in England: evidence from the Health Survey for England. Eur J Public Health 22, 508-513.

27. Edwards S, Murphy C \& Feltbower RG (2006) Changes in the diet of a South Asian trans-migratory population may be associated with an increase in incidence of childhood diabetes. Nutr Res 26, 249-254.

28. Birkbeck JA (1979) New Zealanders and Their Diet: A Report to the National Heart Foundation of New Zealand on the National Dietary Survey 1977. Auckland: Government Printing Office.

29. Howarth C, Parnell W, Birkbeck J et al. (1991) Life in New Zealand Commission Report Volume VI: Nutrition. Dunedin: University of Otago.

30. Russel D, Parnell W, Wilson N et al. (1999) NZ Food: NZ People. Key Results of the 1997 National Nutrition Survey. Wellington: Ministry of Health.

31. Rasanathan K, Craig D \& Perkins R (2006) The novel use of 'Asian' as an ethnic category in the New Zealand Health sector. Ethn Health 11, 211-227.

32. University of Otago \& Ministry of Health (2011) Methodology Report for the 2008/09 New Zealand Adult Nutrition Survey. Wellington: Ministry of Health.

33. Salmond C, Crampton P \& Atkinson J (2007) NZDep2006 Index of Deprivation User's Manual. Wellington: Department of Public Health, University of Otago.

34. World Health Organization (2007) Global Database on Body Mass Index. Geneva: WHO.

35. Cole TJ, Bellizzi MC, Flegal KM et al. (2000) Establishing a standard definition for child overweight and obesity worldwide: international survey. BMJ 320, 1240-1243.

36. WHO Expert Consultation (2004) Appropriate body-mass index for Asian populations and its implications for policy and intervention strategies. Lancet 363, 157-163.

37. Vasudevan D, Stotts AL, Mandayam S et al. (2011) Comparison of BMI and anthropometric measures among South Asian Indians using standard and modified criteria. Public Health Nutr 14, 809-816.

38. Jiang Y-F, Cole T, Pan H-Q et al. (2006) Body mass index percentile curves and cut off points for assessment of overweight and obesity in Shanghai children. World $J$ Pediatr 1, 35-39.

39. Statistics New Zealand (2009) Estimated national ethnic population by age and sex at 30 June 2006 . http://nzdotstat. stats.govt.nz/wbos/Index.aspx?DataSetCode=TABLECODE31\# (accessed May 2013).

40. Beck KL, Kruger R, Conlon CA et al. (2013) Suboptimal iron status and asociated dietary patterns and practices in premenopausal women living in Auckland, New Zealand. Eur J Nutr 52, 467-476.
41. Zhang H, Hsu-Hage BH \& Wahlqvist ML (2002) Longitudinal changes in nutrient intakes in the Melbourne Chinese cohort study. Public Health Nutr 5, 433-439.

42. Scragg R (2010) Asian Health in Aotearoa in 2006-2007; Trends Since 2002-2003. Auckland: Northern DHB Support Agency.

43. Gibson RS (2005) Principles of Nutritional Assessment, 2nd ed. New York: Oxford University Press.

44. Wild S, Roglic G, Green A et al. (2004) Global prevalence of diabetes: estimates for the year 2000 and projections for 2030. Diabetes Care 27, 1047-1053.

45. Misra A, Singhal N, Sivakumar B et al. (2011) Nutrition transition in India: secular trends in dietary intake and their relationship to diet-related non-communicable diseases. J Diabetes 3, 278-292.

46. Radha V \& Mohan V (2007) Genetic predisposition of type 2 diabetes among Asian Indians. Indian J Med Res 125, 259-274.

47. Oza-Frank R \& Venkat Narayan KM (2010) Effect of length of residence on overweight by region of birth and age at arrival among US immigrants. Public Health Nutr 13, 868-875.

48. Gallegos D \& Nasim S (2011) Exploring weight status, dietary intake and acculturation in South Asian women living in Brisbane, Queensland. Nutr Diet 68, 285-290.

49. Institute of Medicine (2000) Dietary Reference Intake: Applications in Dietary Assessment. Washington, DC: National Academy Press.

50. Khokhar S, Pandor A \& Cade J (2001) Eating Habits: Food Preparation and Serving Practices in Ethnic Populations: Critical Review and Assessment of Dietary Survey Requirements. Report to the Food Standards Agency. London: FSA.

51. Khokhar S, Ashkanani F, Garduno-Diaz S et al. (2013) Application of ethnic food composition data for understanding the diet and nutrition of South Asians in the UK. Food Chem 140, 436-442.

52. Ngo J, Gurinovic M, Frost-Andersen L et al. (2009) How dietary intake methodology is adapted for use in European immigrant population groups - a review. Br J Nutr 101, Suppl. 2, S86-S94.

53. Kruger R, Stonehouse W, von Hurst PR et al. (2012) Combining food records with in-depth probing interviews improves quality of dietary intake reporting in a group of South Asian women. Aust N Z J Public Health 36, 135-140.

54. Jonnalagadda SS \& Diwan S (2002) Nutrient intake of first generation Gujarati Asian Indian Immigrants in the US. J Am Coll Nutr 21, 372-380.

55. Hayes L, White M, Unwin N et al. (2002) Patterns of physical activity and relationship with risk markers for cardiovascular disease and diabetes in Indian, Pakistani, Bangladeshi and European adults in a UK population. J Public Health Med 24, 170-178. 